\title{
Comparison of Sagittal FSE T2, STIR, and T1-Weighted Phase-Sensitive Inversion Recovery in the Detection of Spinal Cord Lesions in MS at 3T
}

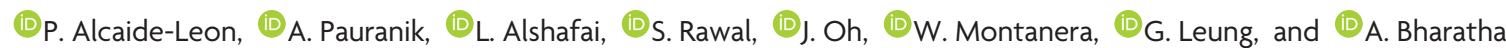

\begin{abstract}
BACKGROUND AND PURPOSE: Determining the diagnostic accuracy of different MR sequences is essential to design MR imaging protocols. The purpose of the study was to compare 3T sagittal FSE T2, STIR, and T1-weighted phase-sensitive inversion recovery in the detection of spinal cord lesions in patients with suspected or definite MS.
\end{abstract}

MATERIALS AND METHODS: We performed a retrospective analysis of 38 patients with suspected or definite MS. Involvement of the cervical and thoracic cord segments was recorded on sagittal FSE T2, STIR, and T1-weighted phase-sensitive inversion recovery sequences independently by 2 readers. A consensus criterion standard read was performed with all sequences available. Sensitivity, specificity, and interobserver agreement were calculated for each sequence.

RESULTS: In the cervical cord, the sensitivity of T1-weighted phase-sensitive inversion recovery (96.2\%) and STIR (89.6\%) was significantly higher $(P<.05)$ than that of FSE T2 $(50.9 \%)$, but no significant difference was found between T1-weighted phase-sensitive inversion recovery and STIR. In the thoracic cord, sensitivity values were $93.8 \%$ for STIR, 71.9\% for FSE T2, and 50.8\% for TI-weighted phase-sensitive inversion recovery. Significant differences were found for all comparisons $(P<.05)$. No differences were detected in specificity. Poor image quality and lower sensitivity of thoracic T1-weighted phase-sensitive inversion recovery compared with the other 2 sequences were associated with a thicker back fat pad.

CONCLUSIONS: The use of an additional sagittal sequence other than FSE T2 significantly increases the detection of cervical and thoracic spinal cord lesions in patients with MS at 3T. In the cervical segment, both STIR and T7-weighted phase-sensitive inversion recovery offer high sensitivity and specificity, whereas in the thoracic spine, STIR performs better than TI-weighted phase-sensitive inversion recovery, particularly in patients with a thick dorsal fat pad.

ABBREVIATION: PSIR = TT-weighted phase-sensitive inversion recovery

$\mathbf{M}^{\mathrm{R}}$ $\mathrm{R}$ imaging of the spinal cord is an important diagnostic technique in MS because the prevalence of spinal cord abnormalities in patients with clinically isolated syndrome is as high as $42 \% .{ }^{1}$ In clinically diagnosed MS, spinal cord involvement reaches $75 \%-92 \%$, depending on the series. ${ }^{2-4}$ The presence of

Received August 25, 2015; accepted after revision November 9

From the Departments of Medical Imaging (P.A.-L., A.P., W.M., G.L., A.B.) and Movement Disorders (J.O.), St Michael's Hospital, Toronto, Ontario, Canada; Department of Medical Imaging (L.A.), University Health Network, Mount Sinai Hospital, Toronto, Ontario, Canada; and Department of Medical Imaging (S.R.), University Health Network, Toronto Western Hospital, Toronto, Ontario, Canada.

Author contributions: guarantors of integrity of entire study: W.M., P.A.-L., A.B.; study concepts/study design or data acquisition or data analysis/interpretation: P.A.-L., L.A., A.P., G.L.; manuscript drafting or manuscript revision for important intellectual content: all authors; approval of the final version of the manuscript: all authors; literature research: P.A.-L.; statistical analysis: P.A.-L.; manuscript editing: P.A.-L., A.B., J.O.

This study was partially funded by Novartis in the form of an educational grant to support the fellowship training position for the first author (P.A.-L.). (Money paid to the institution.) asymptomatic cord lesions contributes to the demonstration of dissemination in space in the McDonald 2010 criteria for MS, and imaging of the spinal cord allows an increase of $18.3 \%$ in the number of patients meeting the diagnostic criteria. ${ }^{5}$ The presence of spinal cord lesions not only facilitates diagnosing MS but is also predictive of conversion to clinically definite MS, especially in patients with nonspinal clinically isolated syndrome who do not fulfill brain MR imaging criteria. ${ }^{6}$ Moreover, spinal cord lesions in MS can occur in isolation in 5\% of patients, particularly in primary-progressive MS. ${ }^{7}$

Spinal cord imaging is challenging because the spinal cord is a small and mobile structure. ${ }^{8}$ In addition, its anatomic location

Please address correspondence to Paula Alcaide-Leon, MD, St Michael's Hospital, Department of Medical Imaging, 30 Bond St, Toronto, ON M5B 1W8, Canada; e-mail: paulaalcaideleon@hotmail.com

E Indicates article with supplemental on-line table.

http://dx.doi.org/10.3174/ajnr.A4656 


\begin{tabular}{|c|c|c|c|c|c|c|c|c|c|c|c|}
\hline Segment & Sequence & Plane & $\begin{array}{l}\text { FOV } \\
(\mathrm{mm})\end{array}$ & $\begin{array}{l}\text { Acquisition } \\
\text { Matrix }\end{array}$ & $\begin{array}{l}\text { Thickness } \\
\text { (mm) }\end{array}$ & TR (ms) & TE (ms) & $\mathrm{TI}$ (ms) & $\begin{array}{l}\text { No. of Signals } \\
\text { Acquired }\end{array}$ & $\begin{array}{l}\text { Acquisition Time } \\
\text { (min:sec) }\end{array}$ & $\begin{array}{l}\text { Parallel } \\
\text { Imaging }\end{array}$ \\
\hline Cervical & PSIR & Sagittal & 220 & $320 \times 224$ & 3 & 2400 & 9.4 & 400 & 2 & $4: 45$ & GRAPPA2 \\
\hline Cervical & STIR & Sagittal & 300 & $448 \times 336$ & 3 & 400 & 51 & 200 & 1 & $5: 46$ & GRAPPA2 \\
\hline Cervical & FSE PD & Sagittal & 220 & $320 \times 288$ & 3 & 2500 & 23 & & 1 & $2: 02$ & None \\
\hline Cervical & FSE T2 & Sagittal & 220 & $384 \times 307$ & 3 & 3500 & 106 & & 2 & $3: 35$ & None \\
\hline Cervical & GE T2 & Transverse & 180 & $256 \times 218$ & 3 & 740 & 17 & & 2 & $4: 25$ & GRAPPA2 \\
\hline Thoracic & PSIR & Sagittal & 330 & $384 \times 269$ & 3 & 2400 & 9.5 & 400 & 2 & $4: 45$ & GRAPPA2 \\
\hline Thoracic & STIR & Sagittal & 330 & $448 \times 336$ & 3 & 4000 & 50 & 200 & 1 & $5: 46$ & GRAPPA2 \\
\hline Thoracic & FSE PD & Sagittal & 330 & $320 \times 288$ & 3 & 2500 & 21 & & 1 & 4:02 & None \\
\hline Thoracic & FSE T2 & Sagittal & 330 & $448 \times 336$ & 3 & 4000 & 96 & & 1 & $4: 26$ & None \\
\hline Thoracic & FSE T2 & Transverse & 180 & $256 \times 176$ & 5 & 6780 & 107 & & 2 & $4: 46$ & None \\
\hline
\end{tabular}

Note:-GE indicates gradient-echo; PD, proton density; GRAPPA, generalized autocalibrating partially parallel acquisition.

makes it prone to ghosting artifacts caused by the heart and great vessels as well as truncation artifacts. $3 \mathrm{~T}$ MR imaging compared with $1.5 \mathrm{~T}$ is more prone to artifacts caused by $\mathrm{B}_{1}$ field inhomogeneity, ${ }^{9}$ susceptibility, vascular pulsation, and chemical shift. ${ }^{10,11}$ In addition, 3T MR imaging has a higher energy deposit within the tissue, resulting in a higher specific absorption rate than lower field scanners. These problems can be partially solved with various technical adjustments and fast (parallel) imaging. ${ }^{12}$

Traditionally, the spinal cord in patients with MS has been imaged by using sagittal and axial FSE T2/proton density sequences. Additional sequences, including STIR ${ }^{13,14}$ and T1 inversion recovery, ${ }^{15}$ have shown promise by increasing lesion visibility, particularly at 3T, in which conventional FSE T2 and proton density images are frequently unsatisfactory. ${ }^{16}$ STIR has proved very useful as a complementary sequence in the detection of MS lesions but cannot be used in isolation due to its lower specificity. ${ }^{13,14}$ Numerous studies have demonstrated the superiority of STIR over T2 at $1.5 \mathrm{~T},{ }^{13,14,17-19}$ and 1 study $^{15}$ also showed the advantages of STIR at $3 \mathrm{~T}$ in the cervical cord. To our knowledge, no studies have been performed in the thoracic cord comparing sagittal FSE T2 and STIR. A recent publication showed the advantages of T1-weighted phase-sensitive inversion recovery (PSIR) for the detection of cervical spinal cord lesions in MS at 3T. ${ }^{16}$ PSIR has been shown to improve lesion localization and boundary definition over STIR in the cervical spinal cord, but it has not been tested in the thoracic cord. ${ }^{16}$

The aim of our study was to compare the sensitivity and specificity of sagittal STIR, PSIR, and FSE T2 in the detection of MS spinal cord lesions at $3 \mathrm{~T}$, in both the cervical and thoracic segments.

\section{MATERIALS AND METHODS \\ Subjects}

Approval of the institutional review board to waive informed consent was obtained for this retrospective study. Inclusion criteria consisted of patients with definite or suspected MS who underwent MR imaging of the spinal cord at 3T, including sagittal STIR, PSIR, and FSE T2 as well as axial T2 sections in our institution from July 1 to October 31, 2013. Exclusion criteria were a final diagnosis of a disease causing spinal cord lesions different from MS and substantial motion artifacts. Spinal MR imaging of 39 consecutive patients was reviewed. One patient with a final diagnosis of Churg-Strauss syndrome was excluded. Thirty-eight patients were included in the study. In 11 patients, only the cervical segment was imaged, and in 12 patients, only the thoracic segment was covered. In 15 patients, both segments were included. This inclusion resulted in 26 cervical and 27 thoracic spinal segments available for evaluation.

\section{MR Imaging Acquisition}

Studies were performed on a 3T MR imaging scanner (Magnetom Skyra; Siemens, Erlangen, Germany) equipped with a 20-channel head-neck coil and a 16-channel spine-array coil. Image parameters are detailed in the Table. No contrast was administered before performing these sequences.

\section{Image Analysis}

Images of all subjects were stripped of patient identifiers and numbered randomly to facilitate blinded analysis. Two readers (P.A.-L. and L.A., neuroradiologists with 6 years' experience in MR imaging) independently evaluated scans for the presence of spinal cord lesions on each spinal cord level on sagittal PSIR, sagittal STIR, and sagittal FSE T2 sequences. To facilitate this task and improve consistency between readers, we drew lines separating the spinal cord levels across the midpoint of the intervertebral disc, parallel to the vertebral endplates as shown in Fig 1C. These reference lines were available during all readings. The upper limit of the $\mathrm{C} 1$ segment was defined by the line connecting the tip of the odontoid and the upper edge of the posterior arch of $\mathrm{C} 1$. The lowest cord segment evaluated was T12, which was limited inferiorly by a line through the T12-L1 disc. Each sequence was evaluated independent of the other sequences so that the readers could not use them to confirm or rule out the presence of a lesion. Readings of different sequences for the same patient were separated by at least a 2 -week interval to avoid memory bias. Disagreements were resolved by consensus. After the 2 independent readings were completed and the disagreements were resolved, an additional consensus reading was performed with all sequences available. This was considered the criterion standard. Combined information contained in sagittal FSE T2, proton density FSE, PSIR, and STIR and in axial gradient-echo T2 of the cervical spine and FSE T2 of the thoracic spine was taken into account to decide which spinal cord segments truly had demyelinating lesions and which were falsely identified as positive for demyelinating lesions due to the presence of artifacts. A lesion was considered a true one if visible on at least 2 sequences, regardless of the imaging plane.

In our experience, PSIR images of the thoracic spine often 

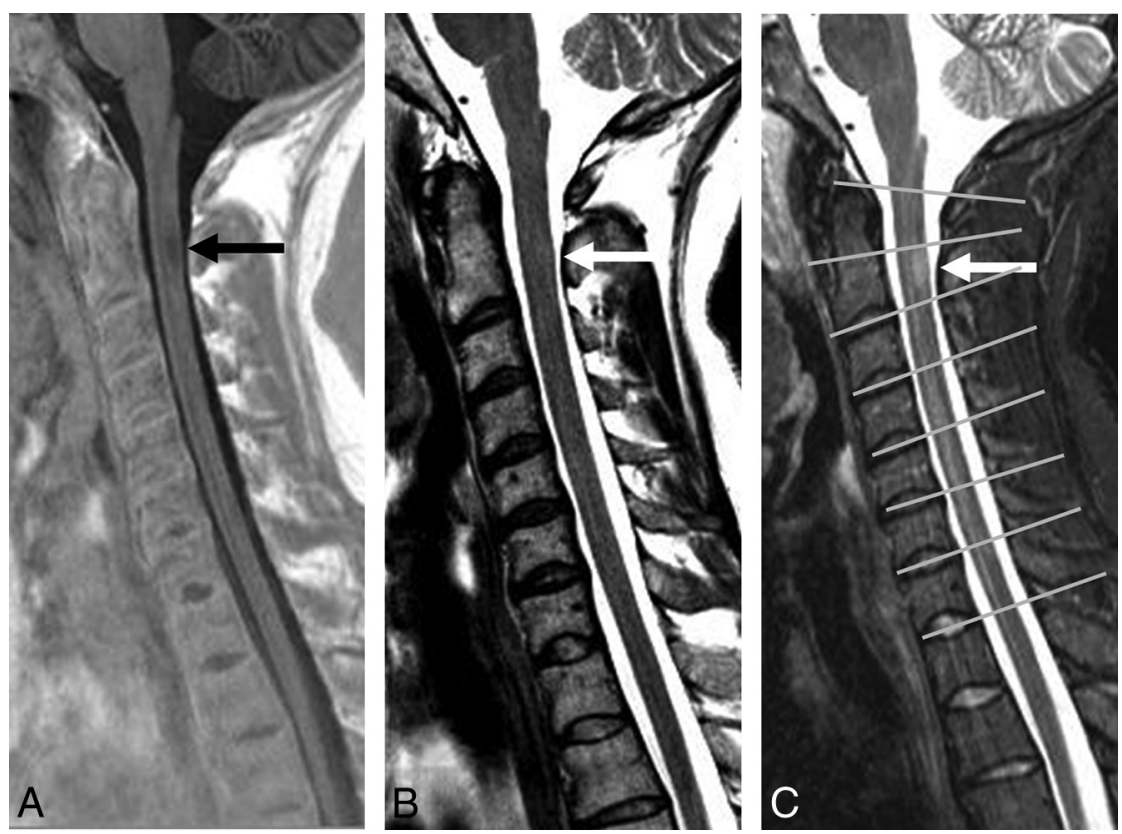

Mann-Whitney $U$ test. The Pearson correlation coefficient was used to assess correlations between the thickness of the back fat pad and the sensitivity of the sagittal PSIR of the thoracic spine.

\section{RESULTS}

Twenty-six female and 12 male subjects were included (mean age, 42.1 years; range, 25-68 years). Of 38 subjects included in the study, 33 had clinically definite MS ( $n=26$, relapsing-remitting MS; $n=3$, primary-progressive MS; $n=$ 3 , secondary-progressive MS; and $n=1$, progressive-relapsing MS). The mean disease duration was 9 years (range, 1-27 years). The remaining 5 subjects included 2 patients with clinically isolated syndrome, 1 patient with radiologically isolated syndrome, and 2 patients with vague sensory symptoms without a definite clinically isolated syndrome.

There were no exclusions due to motion artifacts. As per criterion standard have very poor quality, particularly in patients with a large thoracoabdominal diameter. To confirm our hypothesis that patient size affects PSIR sequence quality in the thoracic spine, we classified images by consensus into 2 groups: good quality or poor quality on the basis of noise and CSF signal saturation. We also measured the thickness of the dorsal back fat pad on PSIR sequences of the thoracic region. This was used as a surrogate marker of the thoracoabdominal diameter, which could not be measured directly on the images. This evaluation was performed by 1 author (P.A.-L.) in the midline at the level of T1-T2, T8-T9, and L1-L2. The mean of the 3 values was calculated. Thicknesses of the back fat pad of patients with goodquality and poor-quality PSIR of the thoracic spine were compared.

\section{Statistical Analysis}

Interrater reliability analysis by the Cohen $\kappa$ coefficient was performed to determine segmental lesion detection consistency among raters. $\kappa$ values were interpreted on the basis of the convention by Landis and Koch. ${ }^{20}$

Estimates of sensitivity and specificity were calculated for each sequence in the cervical and thoracic segments (On-line Table). To perform the calculation, we summated all false-positive, falsenegative, true-positive, and true-negative segments of the different patients. Thus, calculated values of sensitivity and specificity do not represent directly the accuracy of the sequence in diagnosing MS but do represent the accuracy of the sequence in distinguishing an affected and nonaffected segment of the cord. Confidence intervals for sensitivity and specificity were produced with the Wilson score method. ${ }^{21}$ Accuracy of the sequences was also calculated.

Mean thickness of the thoracic back fat pad of the goodquality and poor-quality groups was compared by using the evaluation (consensus reading with all sequences available), spinal cord lesions were present in $84 \%$ of the cervical examinations and in $85 \%$ of the thoracic segments. In 6 patients, the upper thoracic region was not included in the FOV; thus, 8 thoracic segments could not be evaluated. One hundred eighty-two cervical and 316 thoracic spinal cord segments were evaluated. Of these, 106 cervical and 128 thoracic segments were affected by MS lesions. The number of true-positive, true-negative, false-positive, and false-negative spinal cord segments and the sensitivity, specificity, accuracy, and $\kappa$ coefficient of each sagittal sequence are shown in the On-line Table. Comparisons among the number of true-positive, false-negative, and false-positive spinal cord segments of the 3 different sagittal sequences in the cervical and thoracic segments are shown in Fig 2. In the cervical region, the sensitivity of STIR and PSIR was significantly higher than that of FSE T2 $(P<.05)$ (Fig 1$)$. No significant difference was found in sensitivity between PSIR and STIR in the cervical region. In the thoracic region, the sensitivity of STIR was significantly higher than that of FSE T2 and PSIR, and the sensitivity of FSE T2 was significantly higher than that of PSIR $(P<.05)$. Specificities of all cervical and thoracic sagittal sequences studied were very similar, with no significant differences identified. Interobserver agreement was higher for STIR than for FSE T2 and PSIR, in both the cervical and thoracic segments.

At visual inspection, all sequences were rated as good quality except for 10 PSIR thoracic studies that showed inhomogeneous CSF saturation and increased noise (Fig 3). The distribution of the variable "size of the back fat pad" in the good-quality and poorquality groups is shown in Fig $3 E$. Back fat pad thickness in the poor-quality group was significantly higher compared with the high-quality group $(P<.001)$. The mean back fat pad thickness was $13 \mathrm{~mm}(95 \% \mathrm{CI}, 9.9-16.1 \mathrm{~mm})$ in the good-quality group 

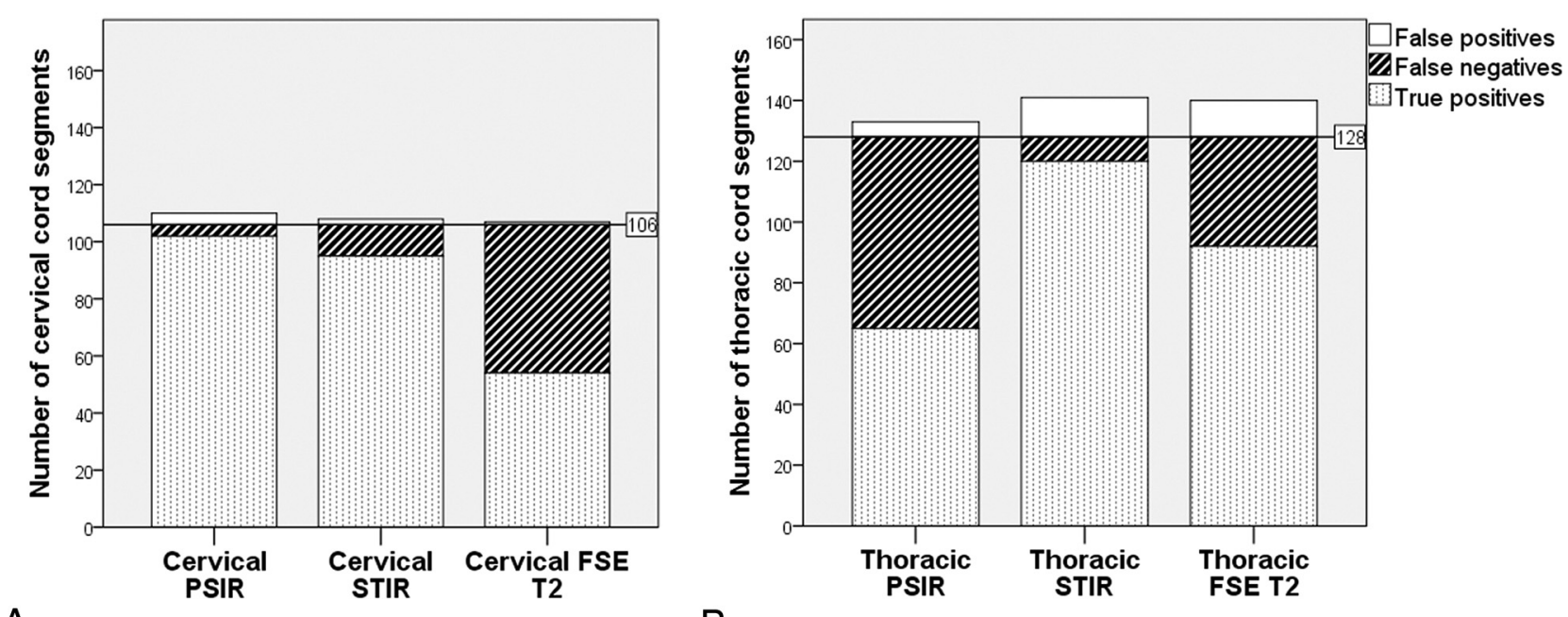

A

B

FIG 2. Stacked bar charts show the total number of true-positive, false-negative, and false-positive spinal cord segments in the cervical ( $A$ ) and thoracic $(B)$ regions on the 3 different sagittal sequences evaluated. The horizontal lines represent the total number of segments truly affected by lesions as per criterion standard evaluation.
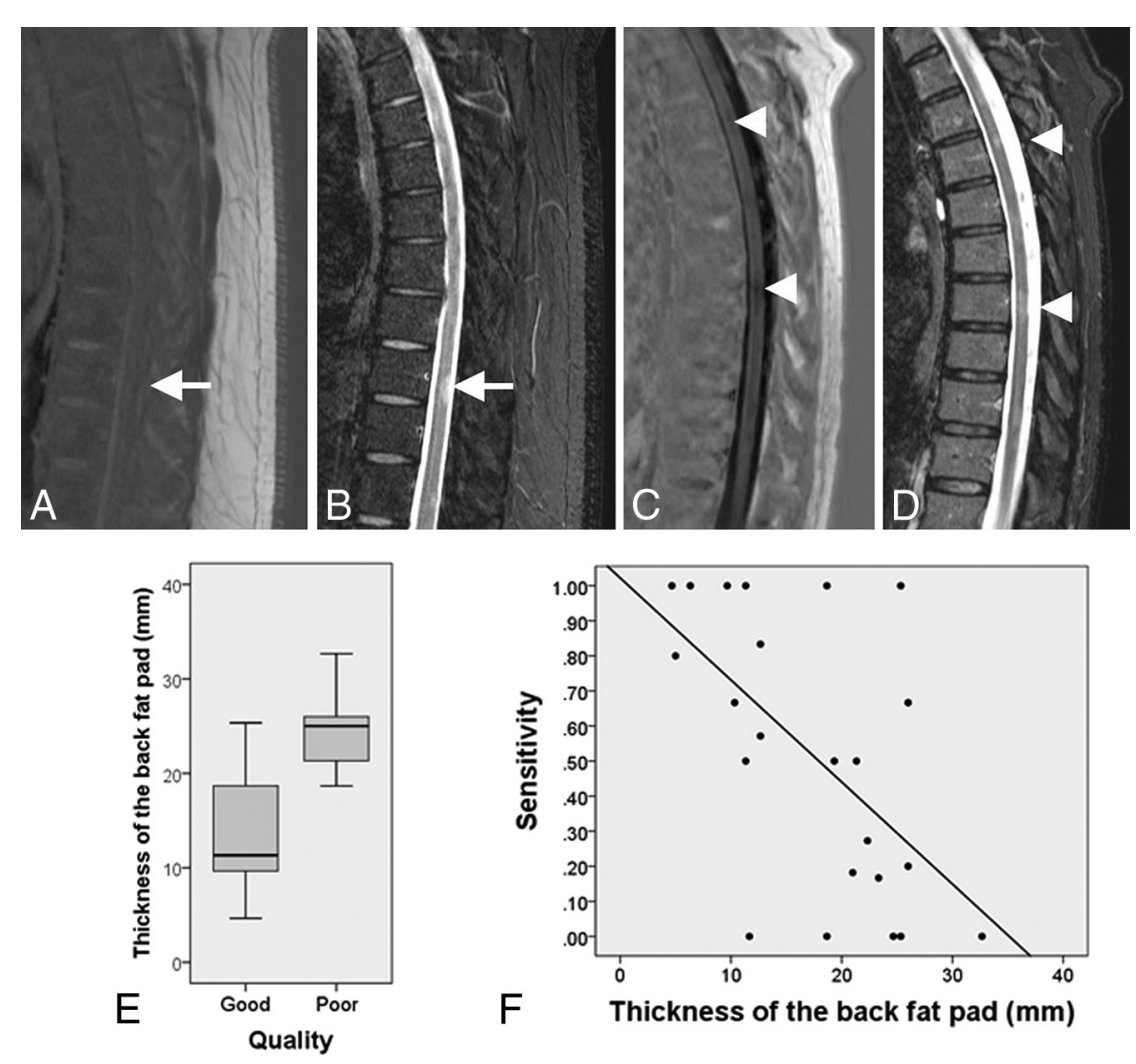

FIG 3. A, Thoracic sagittal PSIR image of a patient with MS with a thick back fat pad shows increased noise and failure of CSF suppression. A demyelinating lesion (white arrow) is clearly identified on STIR $(B)$ but not seen on PSIR $(A)$. Sagittal PSIR $(C)$ and STIR $(D)$ sequences of a slim patient with MS show adequate contrast between the lesions (arrow caps) and the cord and adequate CSF signal suppression on PSIR. E, The boxplot shows the distribution of the variable thickness of the back fat pad in the group of patients with good- and poor-quality thoracic PSIR images. The bottom and the top of the boxes represent the 25th and 75th percentiles, respectively; the median is represented by the horizontal line in the box; and the range corresponds to the whiskers that extend from the box. Patients with poor-quality PSIR sequences of the thoracic spine have a median back fat pad size significantly thicker than that in patients with good-quality images. $F$, The scatterplot shows the negative correlation between the sensitivity of the PSIR sequence of the thoracic segment and the thickness of the back fat pad $(r=-0.53)$. and $24.6 \mathrm{~mm}$ (95\% CI, $21.8-27.3 \mathrm{~mm})$ in the poor-quality group. A moderate negative correlation (Fig $3 F$ ) was found between the thickness of the back fat pad and the sensitivity of the sagittal PSIR sequence of the thoracic spine $(r=$ $-0.58, P=.003)$.

\section{DISCUSSION}

This study shows that lesion detection in the cervical region is lower on sagittal FSE T2 than on PSIR and STIR. The sensitivity of sagittal PSIR in our sample was slightly higher than that of STIR; however, the differences were not significant. In the thoracic spine, lesion detection of STIR was significantly better than that of FSE T2 and PSIR and lesion detection of FSE T2 was significantly better than that of PSIR.

The poor performance of PSIR in the thoracic region appears to be related to the thickness of the back fat pad. We are unsure about the cause of this effect in large patients. One explanation may be the dielectric effect. When the abdominal diameter exceeds the radiofrequency wavelength, the $180^{\circ}$ pulse is not homogeneous through the FOV. Therefore, some areas may be getting an inversion pulse different from $180^{\circ}$. This effect, however, should also be detected on STIR because both are inversion recovery sequences. Another possibility may be a problem in phase unwrapping because the generation of true phase-sensitive images requires phase images to be 
unwrapped first. ${ }^{22}$ The algorithm for phase unwrapping depends on a smooth variation in phase, and this constraint is sometimes not met, depending on the patient's anatomy. A similar problem was found by Riederer et $\mathrm{al}^{23}$ when using a double inversion recovery sequence in the cervical cord. They excluded $10 \%$ of their sample due to artifacts presumably caused by adiposity. We did not observe this problem in the cervical examinations, but we found it in $37 \%$ of our thoracic examinations. We decided not to exclude large patients with poor image quality because our intention was to test sequences as they would be used in the routine clinical practice. Our observation is noteworthy because a sequence that provides suboptimal image quality in $37 \%$ of the patients would not be adequate for routine clinical use; thus, the utility of PSIR to detect thoracic spinal cord lesions may be limited.

Conventionally, sagittal FSE T2 is the most commonly used sequence when imaging the spinal cord in MS. Our results suggest that the use of an additional sagittal sequence can significantly increase the detection of MS lesions in the cervical and thoracic cord without a substantial decrease in specificity. Both sagittal PSIR and STIR perform well in this regard in the cervical spine, but only STIR appears to be adequate in the thoracic region. All the sequences studied have reasonable scanning times and can be implemented in routine clinical practice, but there is a small advantage for cervical PSIR because it is 1 minute shorter than STIR with our acquisition parameters.

We used a segment-based approach to quantify affected spinal cord tissue. With this method, interobserver agreement was moderate for PSIR and FSE T2 of the thoracic spine, substantial for FSE T2 and PSIR of the cervical spine and for STIR of the thoracic spine, and almost perfect for STIR of the cervical spine. Studies comparing lesion detection of different sequences in the spinal cord often had a consensus read ${ }^{16,24}$ and a single read ${ }^{25}$ or did not report $\kappa$ values. ${ }^{14,18,19}$ Of note, our $\kappa$ values using a segment-based approach to quantify spinal cord lesion load are higher than those reported in prior studies ${ }^{13,26}$ that used lesion count; this finding suggests that a segment-based approach may be a more reliable way to quantify lesion burden in the spinal cord in MS.

The results of our study provide useful information for the design of 3T MR imaging protocols in MS. The superiority of STIR over T2 at $1.5 \mathrm{~T}$ has been demonstrated on prior studies. ${ }^{14,19}$ Dietemann et $\mathrm{al}^{25}$ reported that the use of STIR in addition to sagittal T2 in the spinal cord increases the number of MS lesions detected by $46 \%$. Another study by Nayak et al, ${ }^{26}$ which included a variety of scans performed at $1.5 \mathrm{~T}$ and $3 \mathrm{~T}$, showed a $35 \%$ increase in the number of lesions detected using STIR compared with conventional sagittal T2. At 3T, Poonawalla et al ${ }^{16}$ reported improved lesion detection of sagittal STIR over sagittal FSE T2; however, the sample was small $(n=12)$ and no statistical analyses were performed to support the findings. In the study by Philpott and Brotchie, ${ }^{15}$ sagittal STIR showed a statistically significant increase in cervical cord lesion detection compared with FSE T2 at 3T. Our findings, therefore, are in agreement with previous studies at $1.5 \mathrm{~T}$ and $3 \mathrm{~T}$ that have shown that sagittal $\mathrm{T} 2$ alone does not have adequate sensitivity to detect cervical cord lesions (Fig 1), supporting the need to incorporate alternative sequences with higher sensitivity to accurately detect cervical cord lesions in MS. We have also demonstrated the utility of STIR sequences for improving spinal cord lesion detection in the thoracic segment at 3T, which has not been assessed in detail in prior studies.

Our findings support the use of PSIR as another alternative to improve the detection of MS lesions in the cervical spinal cord. In our study, PSIR was not superior to STIR in lesion detection in the cervical spine. Prior studies comparing these 2 sequences include the work by Poonawalla et $\mathrm{al}^{16}$ (cervical STIR versus PSIR) and Philpott and Brotchie ${ }^{15}$ (cervical T1 inversion recovery without phase reconstruction versus STIR); in both cases, samples were very small ( $n=12$ and $n=11$, respectively) and statistical analysis of lesion detection was not performed. Thus, the existing literature does not provide enough evidence regarding the comparison between PSIR and STIR in the cervical cord, though a trend toward PSIR superiority has been observed, as in our study.

Our results raise the question of whether sagittal T2 should be included in the cervical examinations of patients with MS at 3T, given its inferiority compared with other sequences. We have shown the advantage of substituting sagittal T2 with STIR or PSIR in terms of cervical cord lesion detection; however, we have not evaluated the impact of eliminating sagittal T2 in the characterization of nondemyelinating cord pathologies and incidental findings.

This study has a number of limitations. First, we used a segment-based lesion-quantification approach in which the only information recorded was the presence or lack of lesions in each cord segment. Although this method showed substantially better interobserver agreement than lesion count, it has the disadvantage of being insensitive to differential lesion burden within a particular segment. Second, we focused on comparing FSE T2, STIR, and PSIR, but recent studies have demonstrated that double inversion recovery ${ }^{23}$ and T1 MPRAGE ${ }^{24}$ can be applied in the cervical cord of patients with MS; however, their long acquisition time ( $>7$ minutes) limits their application in routine clinical practice. Due to the retrospective nature of our study, we did not have these sequences available for analysis in our study population, but future studies comparing T1-weighted phase-sensitive inversion recovery and T1 MPRAGE with STIR and PSIR would be useful. Third, contrary to prior studies, we did not calculate the body mass index. ${ }^{23}$ This parameter is easy to calculate and would allow rapid prescanning decision-making for STIR versus PSIR.

\section{CONCLUSIONS}

Our results suggest that at 3T, an additional sequence other than sagittal FSE T2 should be incorporated to improve lesion detection in the spinal cord of patients with MS. In the cervical cord, both sagittal STIR and PSIR showed significantly better lesion detection than sagittal FSE T2. In contrast, in the thoracic spinal cord, the sensitivity of PSIR is markedly worse than that of STIR and FSE T2, particularly in large patients, and STIR is clearly superior to FSE T2. These findings should be taken into account when planning sequence protocols to optimize lesion detection in the spinal cord in MS. 


\section{ACKNOWLEDGMENTS}

We would like to thank Bart Schraa, Siemens Application Support, for his help with the technical aspects of this manuscript.

Disclosures: Paula Alcaide-Leon—RELATED: Grant: Novartis.* Sapna RawalUNRELATED: Travel/Accommodations/Meeting Expenses Unrelated to Activities Listed: Association of University Radiologists-GE Radiology Research Academic Fellowship Award, Comments: I am a recipient of the Association of University Radiologists-GE Radiology Research Academic Award for 2015, and the sponsoring body paid for my travel expenses to the Association of University Radiologists meeting in April 2015. Jiwon Oh-UNRELATED: Consultancy: Biogen-Idec, Novartis, Teva, Genzyme, EMD Serono, Roche; Grants/Grants Pending: Biogen-Idec, Genzyme, Multiple Sclerosis Society of Canada*; Payment for Lectures (including service on Speakers Bureaus): EMD Serono, Novartis, Genzyme, Teva, Biogen-Idec; Travel/Accommodations/Meeting Expenses Unrelated to Activities Listed: Genzyme, EMD Serono. General Leung-UNRELATED: Grants/Grants Pending: University of Toronto, * Comments: Internal funding was received for a project for carotid atherosclerosis imaging; Patents (planned, pending or issued): operation of wireless devices through the Faraday Cage, ${ }^{*}$ inhibition of intraplaque hemorrhage using drug therapy*; Other: In-kind support was received from Siemens, Synaptive Medical, VisualSonics/Fujifilm. Aditya Bharatha—RELATED: Grant: Novartis, ${ }^{*}$ Comments: educational grant to support research fellow (first author, P.A.-L.); UNRELATED: Payment for Lectures (including service on Speakers Bureaus): EMD Serono, Novartis, Biogen, Comments: honoraria for educational lectures on MS. *Money paid to the institution.

\section{REFERENCES}

1. Brex PA, O'Riordan JI, Miszkiel KA, et al. Multisequence MRI in clinically isolated syndromes and the early development of MS. Neurology 1999;53:1184-90 CrossRef Medline

2. Ikuta F, Zimmerman HM. Distribution of plaques in seventy autopsy cases of multiple sclerosis in the United States. Neurology 1976;26:26-28 CrossRef Medline

3. Nijeholt GJ, van Walderveen MA, Castelijns JA, et al. Brain and spinal cord abnormalities in multiple sclerosis: correlation between MRI parameters, clinical subtypes and symptoms. Brain 1998; 121(pt 4):687-97 CrossRef Medline

4. Bot JC, Barkhof F, Lycklama à Nijeholt G, et al. Differentiation of multiple sclerosis from other inflammatory disorders and cerebrovascular disease: value of spinal MR imaging. Radiology 2002;223: 46-56 CrossRef Medline

5. Bot JC, Barkhof F, Polman CH, et al. Spinal cord abnormalities in recently diagnosed MS patients: added value of spinal MRI examination. Neurology 2004;62:226-33 CrossRef Medline

6. Sombekke MH, Wattjes MP, Balk LJ, et al. Spinal cord lesions in patients with clinically isolated syndrome: a powerful tool in diagnosis and prognosis. Neurology 2013;80:69-75 CrossRef Medline

7. Thorpe JW, Kidd D, Moseley IF, et al. Spinal MRI in patients with suspected multiple sclerosis and negative brain MRI. Brain 1996; 119(pt 3):709-14 CrossRef Medline

8. Mikulis DJ, Wood ML, Zerdoner OA, et al. Oscillatory motion of the normal cervical spinal cord. Radiology 1994;192:117-21 CrossRef Medline

9. Yang QX, Wang J, Zhang X, et al. Analysis of wave behavior in lossy dielectric samples at high field. Magn Reson Med 2002;47:982-89 CrossRef Medline

10. Phalke VV, Gujar S, Quint DJ. Comparison of 3.0 T versus 1.5 T MR: imaging of the spine. Neuroimaging Clin N Am 2006;16:241-48, ix Medline

11. Fries $P$, Runge VM, Kirchin MA, et al. Magnetic resonance imaging of the spine at 3 Tesla. Semin Musculoskelet Radiol 2008;12:238-52 CrossRef Medline

12. Filippi CG, Carlson M, Johnson JM, et al. Improvements in lumbar spine MRI at $3 \mathrm{~T}$ using parallel transmission. AJR Am J Roentgenol 2012;199:861-67 CrossRef Medline

13. Bot JC, Barkhof F, Lycklama à Nijeholt GJ, et al. Comparison of a conventional cardiac-triggered dual spin-echo and a fast STIR sequence in detection of spinal cord lesions in multiple sclerosis. Eur Radiol 2000;10:753-58 CrossRef Medline

14. Hittmair K, Mallek R, Prayer D, et al. Spinal cord lesions in patients with multiple sclerosis: comparison of MR pulse sequences. AJNR Am J Neuroradiol 1996;17:1555-65 Medline

15. Philpott C, Brotchie P. Comparison of MRI sequences for evaluation of multiple sclerosis of the cervical spinal cord at 3 T. Eur J Radiol 2011;80:780-85 CrossRef Medline

16. Poonawalla AH, Hou P, Nelson FA, et al. Cervical spinal cord lesions in multiple sclerosis: T1-weighted inversion-recovery MR imaging with phase-sensitive reconstruction. Radiology 2008;246:258-64 CrossRef Medline

17. Rocca MA, Mastronardo G, Horsfield MA, et al. Comparison of three MR sequences for the detection of cervical cord lesions in patients with multiple sclerosis. AJNR Am J Neuroradiol 1999;20:1710-16 Medline

18. Campi A, Pontesilli S, Gerevini S, et al. Comparison of MRI pulse sequences for investigation of lesions of the cervical spinal cord. Neuroradiology 2000;42:669-75 CrossRef Medline

19. Mascalchi M, Dal Pozzo G, Bartolozzi C. Effectiveness of the short TI inversion recovery (STIR) sequence in MR imaging of intramedullary spinal lesions. Magn Reson Imaging 1993;11:17-25 CrossRef Medline

20. Landis JR, Koch GG. The measurement of observer agreement for categorical data. Biometrics 1977;33:159-74 CrossRef Medline

21. Julious SA. Two-sided confidence intervals for the single proportion: comparison of seven methods by Robert G. Newcombe, Statistics in Medicine 1998;17:857-872. Stat Med 2005;24:3383-84 Medline

22. Moran PR, Kumar NG, Karstaedt N, et al. Tissue contrast enhancement: image reconstruction algorithm and selection of TI in inversion recovery MRI. Magn Reson Imaging 1986;4:229-35 CrossRef Medline

23. Riederer I, Karampinos DC, Settles M, et al. Double inversion recovery sequence of the cervical spinal cord in multiple sclerosis and related inflammatory diseases. AJNR Am J Neuroradiol 2015;36: 219-25 CrossRef Medline

24. Nair G, Absinta M, Reich DS. Optimized T1-MPRAGE sequence for better visualization of spinal cord multiple sclerosis lesions at 3T. AJNR Am J Neuroradiol 2013;34:2215-22 CrossRef Medline

25. Dietemann JL, Thibaut-Menard A, Warter JM, et al. MRI in multiple sclerosis of the spinal cord: evaluation of fast short-tau inversionrecovery and spin-echo sequences. Neuroradiology 2000;42:810-13 CrossRef Medline

26. Nayak NB, Salah R, Huang JC, et al. A comparison of sagittal short $\mathrm{T} 1$ inversion recovery and $\mathrm{T} 2$-weighted FSE sequences for detection of multiple sclerosis spinal cord lesions. Acta Neurol Scand 2014; 129:198-203 CrossRef Medline 